\title{
Viabilidade do uso de características "dias para um peso específico" em programas de melhoramento genético de bovinos da raça Nelore
}

\author{
Feasibility of using “days to a specific weight” traits in Nellore cattle breeding \\ programs
}

\author{
SANTANA, Bruna Folegatti ${ }^{1 *}$; FONSECA, Ricardo da ${ }^{1}$; MANUEL, Matos ${ }^{2}$; \\ MAMANI, Gerardo Cornelio Mamani ${ }^{3}$; ELER, Joanir Pereira ${ }^{3}$; FERRAZ, José Bento \\ Sterman $^{3}$
}

\footnotetext{
${ }^{1}$ Universidade Estadual Paulistana, Faculdade de Ciências Agrárias e Tecnológicas, Dracena, São Paulo, Brasil.

${ }^{2}$ Universidade Lúrio, Faculdade de Ciências Agrária, Província de Niassa, Unango, Moçambique.

${ }^{3}$ Universidade de São Paulo, Faculdade de Zootecnia e Engenharia de Alimentos, Pirassununga, São Paulo, Brasil

*Endereço para correspondência: bru_fsantana@hotmail.com
}

\section{RESUMO}

O objetivo com este trabalho foi estudar características "dias para um peso específico" em programas de melhoramento genético da raça Nelore, as comparando com características tradicionais como de ganho de peso médio diário. Assim, neste trabalho foram estimados parâmetros genéticos para as características dias para atingir $180 \mathrm{~kg}$ (D180), dias para atingir $300 \mathrm{~kg}$ (D300), ganho de peso pré-desmama (gpmdND), ganho de peso no período da desmama ao sobreano (gpmdDS) para uma população de bovinos da raça Nelore. O banco de dados utilizado é constituído por 60005 animais no arquivo de pedigree e 37234 informações de fenótipos de crescimento. Os dados foram organizados e preparados com auxílio do programa $\mathrm{R}$, e para estimação de componentes de (co)variância realizou-se análises bicaracterísticas com o auxílio do software Wombat. A progênie dos animais foi analisada quanto à variabilidade das diferenças de progênie (Deps) por um coeficiente de variação genético aditivo. As herdabilidades para gpmdND e D180 foram as de maior valor, 0,4 e 0,45 respectivamente. Encontrou-se altas correlações genéticas entre as características, gpmdND e D180 $(0,99)$ e gpmdDS e D300 $(0,95)$. D180 e gpmdDS apresentaram os menores coeficientes de variação. A seleção pelas características pré-desmama produzirá animais que em média levarão o mesmo tempo para chegarem ao peso à desmama. Porém para o peso ao sobreano D300 apresentou vantagem em média de 82,44 dias em relação à gpmdDS. Assim, um índice de seleção que inclua as características D180 e D300 deve produzir os melhores resultados com relação à uniformidade dos selecionados e à velocidade de ganho.

Palavras-chaves: correlações genéticas, ganho por seleção, herdabilidade

\section{SUMMARY}

The objective of this work was to study "days for a specific weight" traits in Nelore breeding programs comparing them with traditional traits such as average daily weight gain. Thus, this study aimed to estimate genetic parameters for days to reach $180 \mathrm{~kg}$ (D180), days to reach $300 \mathrm{~kg}$ (D300), pre-weaning weight gain (gpmdND), weight gain in the weaning period until yearling weight (gpmdDS) traits for a population of Nellore cattle. The used database consists in 60005 animals, and pedigree file information of 37234 growth phenotypes. The data were organized and prepared with the $\mathrm{R}$ program, and the estimation of (co) variance components, bi-character analyzes were performed with software Wombat. The progeny of the animals were analyzed for variability of progeny differences (Deps) by an additive genetic variation coefficient. The heritabilities 
for gpmdND and D180 were the highest, 0.4 and 0.45 , respectively. The analysis demonstrated high genetic correlations between the traits gpmdND and D180 (0.99) and gpmdDS and D300 (0.95). D180 and gpmdDS showed the lowest coefficients of variation. Selection by the pre-weaning traits will produce animals that will take, on average, the same time to reach the weaning weight. However, for the D300 weight, the average gain was 82.44 days in relation to gpmdDS. Thus, a selection index that includes the traits D180 and D300 should produce the best results regarding the uniformity of the selected ones and the speed of gain.

Keywords: genetic correlations, heritability, selection gains

\section{INTRODUÇÃO}

O Brasil apresenta o maior rebanho comercial e possui aproximadamente 215,2 milhões de cabeças, sendo $80 \%$ do rebanho composto por animais zebuínos (IBGE, 2016). A raça nelore é a mais numerosa estando presente em 90\% dentre o rebanho zebuíno (FARIA et al., 2015). Devido à importância da raça, tem sido desenvolvido em todo o país programas de melhoramento genético e características relacionadas à velocidade de crescimento são incluídas como critério de seleção. Para eficiência destes programas é necessário o conhecimento de parâmetros genéticos de características de valor econômico. A velocidade de crescimento expressa como ganho de peso médio diário em diferentes períodos ou como pesos ajustados para certas idades, são utilizados como critério de seleção há várias décadas, e já se tem conhecimento sobre os parâmetros genéticos destas características em bovinos (FRIES et al., 1996).

Entretanto, foi sugerido por Fries et al. (1996) a característica "dias para um peso específico", com o objetivo de selecionar animais para velocidade de ganho até um peso estratégico definido e com maior uniformidade de progênie para bovinos de corte.

Garneiro et al. (2001) comparando critérios de seleção, em bovinos Nelore, estimou parâmetros genéticos para características "pesos padronizados" e "número de dias para ganhar um peso específico" observando vantagens na seleção pelo uso dos tradicionais "pesos padronizados" nas respostas relacionadas à crescimento. Estudos como de Tanaka et al. (2009) para bovinos da raça Guzerá, compararam a seleção com base no "ganho médio diário de peso na prédesmama" (GMD) e no "número de dias para ganhar 160kg" (D160) nesta fase, chegando à conclusão que ambas tem potencial para responder à seleção para precocidade de crescimento. Porém, a seleção para D160 favorece touros que produzem progênie com desempenho superior e menos variável. Apesar desta característica, "dias para um peso específico", estar presente em importantes catálogos de venda de sêmen bovinos e do desenvolvimento de estudos neste sentido, não se chegou a um consenso sobre a vantagens de sua utilização. $O$ objetivo deste trabalho foi estudar a viabilidade do uso de características "dias para um peso específico" em programas de melhoramento genético de bovinos da raça Nelore.

\section{MATERIAL E MÉTODOS}

Os dados utilizados neste estudo foram provenientes da Fazenda Mundo Novo. O banco de dados é constituído por 60005 animais no arquivo de pedigree e 
possui 37234 informações de fenótipos de crescimento.

A organização e a preparação dos dados foram realizadas com o auxílio do programa do programa $\mathrm{R}$ (R CORE TEAM, 2014).

Para este estudo foram consideradas as seguintes características:

Dias para atingir $180 \mathrm{~kg}$ (D180); Dias para atingir $300 \mathrm{~kg}$ (D300); Ganho de peso pré-desmama, do nascimento aos 205 dias (gpmdND); Ganho de peso da pós-desmama aos 540 dias (gpmdDS). Os grupos de contemporâneos (GC) foram definidos a partir da análise dos efeitos ambientais registrados. Os efeitos disponíveis para análise foram: fazenda, sexo, grupo de manejo e estação. Cada GC foi contemplado com no mínimo 5 animais.

Informações sobre o banco de dados e sua estrutura podem ser observadas na Tabela 1. Em relação às características em estudo, a Tabela 2 apresenta um resumo de suas distribuições.

Tabela 1. Estrutura dos dados no arquivo estudado

\begin{tabular}{lc}
\hline Número de animais & 60005 \\
Número de touros & 885 \\
Número de vacas & 17388 \\
Número de GC & 1120 \\
\hline
\end{tabular}

Tabela 2. Informações sobre as características no arquivo estudado

\begin{tabular}{lcccc}
\hline Item & Média & DP & Min & Máx \\
\hline gpmdND & 691,00 & 113,83 & 401,5 & 996 \\
gpmdDS & 360,00 & 82,68 & 222,5 & 598,8 \\
D180 & 270,00 & 38,79 & 170,2 & 360 \\
D300 & 945,00 & 190,26 & 411,6 & 1299 \\
\hline
\end{tabular}

Média: média das características em estudo; DP: Desvio Padrão das características em estudo; Min: Valor mínimo encontrado para cada característica; Máx: Valor máximo encontrado para característica.

A característica gpmdND foi calculada com a diferença entre o peso da desmama e o peso do nascimento dividido pelo número de dias dentro do período. Já gpmdDS foi a diferença entre o peso ao sobreano e o peso da desmama dividido pelo número de dias dentro do período.

D180 e D300 foram calculadas da seguinte forma:

$$
\begin{aligned}
& D 180=\frac{180}{g p m d N D} \\
& D 300=\frac{300}{g p m d D S}
\end{aligned}
$$

Em todas as análises foi utilizado o modelo animal. Os três modelos que foram considerados, diferenciavam-se pelos efeitos aleatórios. O primeiro modelo incluiu o efeito genético aditivo direto, como aleatório, além dos efeitos fixos de GC. O segundo modelo incluiu, além dos efeitos do modelo anterior, o efeito aleatório do ambiente materno. $\mathrm{O}$ terceiro modelo compreende os efeitos genéticos diretos e maternos e o efeito de ambiente materno. Através do teste de verossimilhança o modelo mais adequado foi definido.

Os três modelos estudados em análise bi característica são como segue:

Modelo 1: $Y=X \beta+Z a+e$

Modelo 2: $Y=X \beta+Z a+\wp+e$

Modelo 3: $Y=X \beta+Z a+M m+$

$W \wp+e$, 
Em que: Y é o vetor das observações; $\beta$, $\alpha, m, \wp$ são os vetores dos efeitos fixos, genético aditivo, materno e ambiente materno, respectivamente; $\mathrm{X}, \mathrm{Z}, \mathrm{M}, \mathrm{W}$, são as matrizes de incidência relacionadas aos respectivos efeitos; e é o vetor dos resíduos aleatórios associados a cada observação.

As pressuposições em relação aos componentes foram:

$\mathrm{E}(\mathrm{y})=\mathrm{X} \beta$

$\mathrm{E}(\mathrm{a})=0, \mathrm{E}(\mathrm{m})=0, \mathrm{E}(\mathrm{c})=0$ e $\mathrm{E}(\mathrm{e})=0$.

Em que: $\mathrm{A}=$ matriz de parentesco genético aditivo; $I_{n v}$ e $I_{n b}=$ matrizes identidade de dimensão número de mães $(n v)$ e total de observações $(n b)$; $\sigma_{a}^{2}=$ variância genética aditiva direta, $\sigma_{m}^{2}$ variância genética aditiva materna; $\sigma^{2}{ }_{c}=$ variância de ambiente materno e $\sigma_{e}^{2}=$ variância residual.

Dado os resultados do teste da razão de verossimilhança, o Modelo 1 foi $o$ escolhido para estimação de parâmetros genéticos e predição de valores genéticos aditivos entre as características gpmdDS e D300, e o Modelo 3 para as características gpmdND e D180.

Para estimação de componentes de (co)variância, análises uni características foram inicialmente realizadas com 0 objetivo de se obter valores iniciais mais adequados para os componentes de variâncias das análises bi característica.

Os componentes de (co)variância estimados nas análises bi características foram utilizados para a predição dos valores genéticos aditivos.

Todas as análises foram realizadas utilizando o método de máxima verossimilhança restrita (REML) por meio do software Wombat (MEYER., 2007). O critério de convergência utilizado foi de $10^{-6}$.

Para comparar a uniformidade das progênies entre os reprodutores para as características estudadas, a seguinte estatística foi calculada utilizando-se as DEPs calculadas préviamente:

$$
\gamma_{\mathrm{a}_{\mathrm{i}}}=\frac{\sigma^{\wedge} \mathrm{a}_{\mathrm{i}}}{\mathrm{x}_{\mathrm{i}}}
$$

em que,

$\gamma_{\mathrm{a}_{\mathrm{i}}}=$ Coeficiente de variação genético aditivo ou fenotípico para a característica $\mathrm{i} ; \mathrm{k}=\mathrm{a}$ ou $\mathrm{p}$; em que $\mathrm{a}=$ componente genético aditivo e $\mathrm{p}=\mathrm{o}$ componente fenotípico:

$\sigma^{\wedge}{ }_{a_{i}}=$ estimativa do componente de variância genética aditiva ou fenotípica da característica $\mathrm{i} ; \bar{x}_{\mathrm{i}}=$ média da população para a característica $i$.

A estatística $\gamma$ foi calculada para a progênie de todos os reprodutores da população.

Foi realizado o cálculo de ganho genético para cada característica por geração, seguindo a fórmula:

$$
\Delta G / G=h^{2} \times i \times \sigma_{p}^{\wedge}
$$

em que,

$\Delta G / G=$ ganho genético por geração; $h^{2}=$ herdabilidade direta; $i=$ intensidade seletiva; $\sigma_{p}=$ desvio padrão fenotípico.

\section{RESULTADOS E DISCUSSÃO}

As estimativas dos coeficientes de herdabilidade e componentes de variância são apresentados na Tabela 3 . Para os efeitos diretos, a herdabilidade para gpmdND e D180 foram mais altas, e assim, para programas de melhoramento da raça inicialmente essas características seriam as que apresentariam maiores potenciais de respostas à seleção. As estimativas dos coeficientes de herdabilidade maternos foram baixas para as características. 
Tabela 3. Estimativas de variância genética aditiva $\left(\sigma^{a^{2}}\right)$, materna $\left(\sigma^{m^{2}}\right)$, de ambiente permanente $\left(\sigma^{p e^{2}}\right)$ e residual $\left(\sigma^{\wedge} \mathrm{e}^{2}\right)$, herdabilidade direta $\left(h^{a^{2}}\right)$ e herdabilidade materna $\left(h^{m^{2}}\right)$ das análise bi características de ganho de peso diário, e de dias para um determinado peso

\begin{tabular}{lcccccc}
\hline Características & $\sigma^{a^{2}}$ & $\sigma^{m^{2}}$ & $\sigma^{p e^{2}}$ & $\sigma^{e^{2}}$ & $h^{a^{2}}$ & $h^{m^{2}}$ \\
\hline gpmdND & 267063,33 & 23367,33 & 0,00 & 382380,00 & $0,40(0,006)$ & $0,03(0,006)$ \\
gpmdDS & 193180,00 & - & - & 766640,00 & $0,20(0,003)$ & - \\
D180 & 213430,00 & 20429,33 & 0,01 & 243140,00 & $0,45(0,011)$ & $0,04(0,006)$ \\
D300 & 357310,00 & - & - & 2356366,67 & $0,13(0,001)$ & - \\
\hline
\end{tabular}

gpmdND = ganho de peso médio diário no período do nascimento à desmama (em gramas); gpmdDS = ganho de peso médio diário no período da desmama ao sobreano (em gramas); D180 = dias para atingir $180 \mathrm{Kg}$; D300 = dias para atingir $300 \mathrm{Kg}$.

Laureano et al. (2011) estimando parâmetros genéticos na raça Nelore encontrou herdabilidades para o ganho de peso pré-desmama e da desmama ao sobreano de 0,21 e 0,23 respectivamente, se assemelhando com a herdabilidade encontrada neste trabalho para gpmdDS, entretanto se diferindo significativamente em gpmdNS. Características "número de dias para ganhar $160 \mathrm{~kg}$ " do nascimento à desmama (D160) e "número de dias para atingir $400 \mathrm{~kg}$ " (D400) do nascimento ao sobreano em estudos desenvolvidos anteriormente com bovinos apresentaram herdabilidades baixas a médias $(0,09$ a 0,40) (ALBUQUERQUE \& FRIES., 1996; FRIES et al., 1996; ORTIZ PEÑA., 1998; GARNERO et al., 2001; MUNIZ et al., 2005, SOUZA et al., 2008). As características D180 e D300 se mostraram dentro do intervalo apresentado pelos autores citados. Com objetivo de peso à desmama e ao sobreano estabelecidos em $180 \mathrm{Kg}$ e $300 \mathrm{Kg}$, respectivamente, esta comparação se dá por se tratar de características que medem em dias o tempo para se atingir um peso determinado previamente.

$\mathrm{Na}$ Tabela 4, altas correlações genéticas podem ser observadas entre as características, gpmdND e D180 $(0,99)$ e gpmdDS e D300 (0,99). A análise desses resultados sugere que as características não devem ser usadas simultaneamente em índices de seleção, uma vez que, pelas altas correlações genéticas encontradas, a seleção para uma delas causará uma resposta correlacionada de magnitude semelhante na outra. A característica gpmdDS, por possuir uma maior herdabilidade (Tabela 1), poderia ser utilizada em um índice de seleção com a finalidade de se conseguir ganhos genéticos diretos para essa, bem como ganhos indiretos em D300. Assim como se pode esperar um maior incremento nos ganhos pela seleção da característica D180 e consequentemente respostas em gpmdND.

Os coeficientes de variação genética apresentados na Tabela 5 mostram que no rebanho em estudo selecionando-se as progênies de touros pelas características avaliadas e observandose o quesito uniformidade, as progênies selecionadas pelas características gpmdDS e D180 são as que apresentam maiores vantagens. 
Tabela 4. Estimativas de correlações genéticas (acima da diagonal) e correlações fenotípicas (abaixo da diagonal) entre ganhos de peso diários, e dias para um determinado peso

\begin{tabular}{lcccc}
\hline Item & gpmdND & gpmdDS & D180 & D300 \\
\hline gpmdND & $*$ & 0,2105 & 0,9923 & 0,4661 \\
gpmdDS & 0,3585 & $*$ & 0,2011 & 0,9558 \\
D180 & 0,9722 & 0,3455 & $*$ & 0,2246 \\
D300 & 0,1011 & 0,7323 & 0,2575 & $*$ \\
\hline
\end{tabular}

gpmdND = ganho de peso médio diário no período do nascimento à desmama (em gramas); gpmdDS = ganho de peso médio diário no período da desmama ao sobreano (em gramas); D180 = dias para atingir $180 \mathrm{Kg} ; \mathrm{D} 300=$ dias para atingir $300 \mathrm{Kg}$.

Tabela 5. Coeficiente de variação genético aditivo (número de indivíduos utilizado no cálculo), para as características de ganho de peso diário, e de dias para um determinado peso entre a progênie de todos os touros para cada característica

\begin{tabular}{lc}
\hline Item & CVGA \\
\hline gpmdND & $1,241(11481)$ \\
gpmdDS & $0,427(1313)$ \\
D180 & $0,604(11590)$ \\
D300 & $2,487(3418)$ \\
\hline CVGA = coeficiente de variação genético
\end{tabular}
aditivo; gpmdND = ganho de peso médio diário no período do nascimento à desmama (em gramas); gpmdDS = ganho de peso médio diário no período da desmama ao sobreano (em gramas); D180 = dias para atingir $180 \mathrm{Kg}$; D300 $=$ dias para atingir $300 \mathrm{Kg}$.

A Tabela 6 mostra o tempo esperado para se alcançar o peso ao desmame pelas características gpmdND e D180 e também o tempo para que se chegar ao peso ao abate pelas características gpmdDS D300.

Para se chegar aos valores de quantidade de dias primeiramente foi calculado os ganhos que as características trariam, e então acrescidos às médias fenotípicas de cada característica. Para intensidade seletiva $\mathrm{i}=1$.
Tabela 6. Tempo para se alcançar o peso a desmama pelo uso das características para prédesmama

\begin{tabular}{lc}
\hline Item & Tempo 180 Kg \\
\hline gpmdND & 200,81 \\
D180 & Tempo 300 Kg \\
\hline \multicolumn{2}{c}{314,23} \\
\hline gpmdDS & 231,79 \\
D300 & \\
\hline gpmdND = ganho de peso médio diário no \\
período do nascimento à desmama (em \\
gramas); D180= dias para atingir 180 Kg; \\
gpmdDS = ganho de peso médio diário no \\
período da desmama ao sobreano (em \\
gramas); D300= dias para atingir 300 Kg.
\end{tabular}

Pela seleção das características gpmdND e D180 se espera que o tempo em ambas para se atingir os $180 \mathrm{~kg}$ seja de aproximadamente 200 dias. Logo, neste ponto a seleção na pré-desmama tanto de uma como da outra característica trará resultados semelhantes.

Entretanto, o tempo para se atingir os $300 \mathrm{~kg}$ (com a contagem iniciada após se atingir $180 \mathrm{~kg}$ ), a seleção de D300 mostrou vantagem em 82,44 dias em comparação à gpmdDS.

Peripolli et al. (2016), encontrou para Nelore, dentro do modelo de criação totalmente à pasto, Custo/Animal/Mês seria R $\quad 17,51$. Fazendo uma média de custo por animal/dia o valor será de $\mathrm{R} \$ 0,58$. 
Considerando 100 animais da raça Nelore, selecionados por D300 e atingindo o peso ao sobreano 82,44 dias mais cedo em relação aos selecionados por gpmdDS, o custo neste período de tempo que poderia ser evitado será de aproximadamente de $\mathrm{R} \$ 4781,50$.
Na Figura 1 são apresentados valores de ganho genético esperado. Esses ganhos foram calculados com diferentes intensidades de seleção, revelando o quanto se pode obter de melhora das características após uma geração.

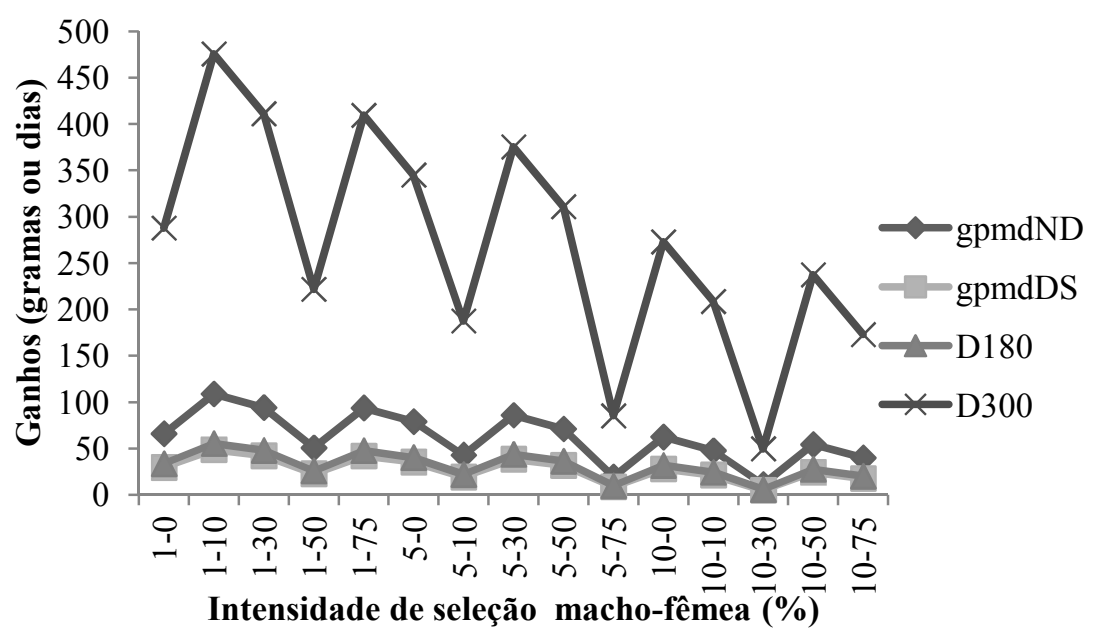

Figura 1. Ganhos genéticos das características em estudo de acordo com as diferentes intensidades de seleção, sendo gpmdND = ganho de peso médio diário no período do nascimento à desmama (em gramas); D180 = dias para atingir $180 \mathrm{Kg}$; gpmdDS $=$ ganho de peso médio diário no período da desmama ao sobreano (em gramas); D300 = dias para atingir $300 \mathrm{Kg}$

Sabe-se que quanto maiores as intensidades de seleção maiores serão os progressos genéticos. Com as intensidades $1 \%$ e $10 \%$ para machos e fêmeas, respectivamente, espera-se que em uma geração a seleção pelas características gpmdND e pela gpmdDS produza um incremento em média de 108,92 e 48,8 gramas diárias, respectivamente. Também que D180 diminua em média 55,55 dias para se alcançar o peso à desmama enquanto D300 diminuiria 475,47 dias em média para se alcançar $300 \mathrm{Kg}$. Estes valores possuem relação com as médias fenotípicas das características, de gpmdND a média é 694,1 gramas/dia, de gpmdDS 360,3 gramas/dia, D180 268,5 dias e a média fenotípica desta população para D300 é de 946,5 dias.

Observa-se que as características D180 e gpmdND apresentam altas correlações genéticas, logo ao selecionar uma se obterá respostas de grande magnitude na outra, assim como acontece com D300 e gpmdDS. Sendo a uniformidade um fator relevante, deve-se buscar alcança-la na pré-desmama pela característica D180. Na pós-desmama deve-se explorar a característica D300 que mostrou-se vantajosa ao possibilitar que o peso ao sobreano seja atingido mais precocemente, trazendo benefícios econômicos evidentes. 


\section{AGRADECIMENTOS}

À Fapesp, processo 2014/07566-2.

\section{REFERÊNCIAS}

ALBUQUERQUE, L.G.; FRIES, L.A. Conseqüências genéticas de selecionar pelo numerador ou contra o denominador do GMD. In: CONGRESSO BRASILEIRO DAS RAÇAS ZEBUÍNAS, 2, 1996, Uberaba. Anais... Uberaba: ABCZ, 1996

FARIA, C.U.; ANDRADE, W.B.F.; PEREIRA, C.F.; SILVA, RP.; LÔBO, R.B. Análise bayesiana para características de carcaça avaliadas por ultrassonografia de bovinos da raça nelore mocho, criados em bioma cerrado. Ciência Rural, v.45, p.317$322,2015$.

FRIES, L.A.; BRITO, F.V.; ALBUQUERQUE, L.G. Possíveis conseqüências de seleção para incrementar pesos às idades padrão vs. reduzir idades para produzir unidades de mercado. In: REUNIÃO ANUAL DA SOCIEDADE BRASILEIRA DE ZOOTECNIA, 33., 1996, Fortaleza. Anais... Fortaleza: Sociedade Brasileira de Zootecnia, 1996. p. 310-312.

GARNERO, A.V.; LÔBO, R.B.; BEZERRA, L.A.F; OLIVEIRA, H.N. Comparação entre Alguns Critérios de Seleção para Crescimento na Raça Nelore. Revista Brasileira de Zootecnia, v.30, n.3, p.714-718, 2001.

\section{INSTITUTO BRASILEIRO DE} GEOGRAFIA E ESTATÍSTICA - IBGE. Produção da pecuária municipal. 2016. Dinponível em: $<$ http://saladeimprensa.ibge.gov.br/notici as.html? view=noticia\&id=1 \&idnoticia=3 268\&busca $=1 \& \mathrm{t}=$ ppm-rebanho-bovinoalcanca-marca-recorde-215-2-milhoescabecas-producao-leite>. Acesso em: 1 out. 2016.

LAUREANO, M.M.M.; BOLIGON, A.A.; COSTA, R.B; FORNI, S.; SEVERO, J.L.P.; ALBUQUERQUE, L.G. Estimativas de herdabilidade e tendências genéticas para características de crescimento e reprodutivas em bovinos da raça Nelore. Arquivo Brasileiro de Medicina Veterinária e Zootecnia, v.63, n.1, p.143-152, 2010.

MEYER, K.. WOMBAT A tool for mixed model analyses in quantitative genetics by restricted maximum likelihood. Journal of Zhejiang University: Science B, v.8, n.11, p.815821, 2007.

MUNIZ, C.A.S.D.; CAVALHEIRO, R.; FRIES, L.A.; QUEIROZ, S.A. Dois Critérios de Seleção na Pré-Desmama em Bovinos da Raça Gir. 1. Estimativas de parâmetros genéticos. Revista Brasileira de Zootecnia, v.34, n.3, p.807-815, 2005.

PERIPOLLI, E.; OLIVEIRA, M.A.L.; BALDI, F.; PEREIRA, A.S.C.; VERCESI, A.E. e ALBUQUERQUE, L.G. Valores econômicos para sistemas de recria e engorda de bovinos Nelore e cruzado. Arch Zootec, v.60, p.145-154, 2016.

ORTIZ PEÑA, C.D. Análise de critérios de seleção para precocidade sexual e de crescimento de bovinos da raça Nelore, no Paraguai. 1998. 143p. Dissertação (Mestrado) - Faculdade de Ciências Agrárias e Veterinárias, Universidade Estadual Paulista, Jaboticabal. 
R DEVELOPMENT CORE TEAM. R:

A language and environment for statistical computing. Vienna: R Foundation for Statistical Computing, 2014. Disponível em:

$<$ http:www.Rproject.org $>$. Acesso em: set. 2016.

SOUZA, J.C.; SILVA, L.O.C.; SIMÕES, G.H.; MOSER, J.T.; OSTAPECHEN, J.; PINTO, P.H.N.; RUVIEIRO, V.; MALHADO, C.H.M.; FILHO, P.B.F.; FREITAS, J.A.; SERENO, J.R.B. Tendências ambientais e genéticas para características produtivas de bovinos da raça Nelore. Archivos

Latinoamericanos de Producción

Animal, v.6, n.2, p.85-90, 12, 2011.

TANAKA, A.L.R.; CAVALHEIRO, R.; FRIES, L.A.; QUEIROZ, S.A.

Comparação de critérios de seleção para precocidade de crescimento em bovinos da raça Guzerá. Revista Brasileira de Zootecnia, v.38, n.2, p.284-291, 2009.

Data de recebimento: 03/01/2017

Data de aprovação: 06/04/2017 